\title{
Intelligent Optimized Combined Model Based on GARCH and SVM for Forecasting Electricity Price of New South Wales, Australia
}

\author{
Yi Yang, ${ }^{1}$ Yao Dong, ${ }^{2}$ Yanhua Chen, ${ }^{1}$ and Caihong $\mathrm{Li}^{1}$ \\ ${ }^{1}$ School of Information Science and Engineering, Lanzhou University, Lanzhou 730000, China \\ ${ }^{2}$ School of Mathematics and Statistics, Lanzhou University, Lanzhou 730000, China \\ Correspondence should be addressed to Yao Dong; dongsky2005@gmail.com
}

Received 14 January 2014; Accepted 26 February 2014; Published 6 April 2014

Academic Editor: Haiyan Lu

Copyright (c) 2014 Yi Yang et al. This is an open access article distributed under the Creative Commons Attribution License, which permits unrestricted use, distribution, and reproduction in any medium, provided the original work is properly cited.

\begin{abstract}
Daily electricity price forecasting plays an essential role in electrical power system operation and planning. The accuracy of forecasting electricity price can ensure that consumers minimize their electricity costs and make producers maximize their profits and avoid volatility. However, the fluctuation of electricity price depends on other commodities and there is a very complicated randomization in its evolution process. Therefore, in recent years, although large number of forecasting methods have been proposed and researched in this domain, it is very difficult to forecast electricity price with only one traditional model for different behaviors of electricity price. In this paper, we propose an optimized combined forecasting model by ant colony optimization algorithm (ACO) based on the generalized autoregressive conditional heteroskedasticity (GARCH) model and support vector machine (SVM) to improve the forecasting accuracy. First, both GARCH model and SVM are developed to forecast short-term electricity price of New South Wales in Australia. Then, ACO algorithm is applied to determine the weight coefficients. Finally, the forecasting errors by three models are analyzed and compared. The experiment results demonstrate that the combined model makes accuracy higher than the single models.
\end{abstract}

\section{Introduction}

It is a challenging task and significant role to forecast electricity price in competitive electricity market. However, electricity price has distinct characteristics from other commodities, which is due to its nonlinearity, nonstationarity, time variance, and uncertain bidding strategy of the market participants. All these characteristics can be attributed to the following reasons, which distinguish electricity from other commodities: (i) nonstorable nature of electrical energy, (ii) the requirement of maintaining constant balance between demand and supply, (iii) inelastic nature of demand over short time period, and (iv) oligopolistic generation side [1]. According to an accurate daily price forecasting in the electricity market, the power suppliers can reduce the cost of electricity production, optimize the allocation of resources, reduce the uncertainty of production, maximize profits, and ensure the dominant position in the market competition. At the same time, the consumers can also make a plan to maximize their utilities and minimize their costs using the electricity purchased from the pool or using self-production to protect themselves against high prices.

In the current power forecasting researches, the forecasting of electricity demand and price has emerged as one of the major research fields in electrical engineering [2]. A lot of researchers and academicians are engaged in the activity of developing tools and algorithms for load and price forecasting [1]. Whereas load forecasting has reached advanced stage of development and load forecasting algorithms with mean absolute percentage error (MAPE) below 3\% are available [3, 4], price-forecasting techniques, which are being applied, are still in their early stages of maturity. Although a few attempts have already been made in this direction, only qualitative aspects of price forecasting have been addressed. So the importance and complexity of electricity price forecasting motivate many researches in this area, especially in the recent years [5]. 
The electricity price prediction can be classified into two categories. One is the detailed market simulation that requires plenty of market information. The most popular approach is the artificial neural network (ANN) technique. ANN technique which possesses excellent robustness and error tolerance is an effective way to solve the complex nonlinear mapping problem. But the ANN contains a great many parameters. These parameters are always judged by experience, so the model is hard to be established [6]. Besides, it has been observed that while the neural network $(\mathrm{NN})$ gives small error for training patterns, the error for testing patterns is usually of larger order [7]; in other words, when this method is applied to practical system, the accuracy is not good.

The other forecasting technology refers to some mathematical approaches without a thorough market modeling, but which attempt to discover the relation between some known inputs and the electricity price. Arciniegas and Arciniegas Rueda [8] applied a Takagi-Sugeno-Kang (TSK) fuzzy inference system to forecast the one-day-ahead real-time peak price of the Ontario Electricity Market. TSK's improvements in forecasting with respect to ANN and ARMAX are above $10 \%$, and it has considerable value to forecast one-day-ahead peak price. Fuzzy system does not need to establish a precise mathematical model, but its adaptive capacity is limited, and a steady-state error may cause oscillations. Lei and Feng [9] proposed a novel grey model to forecast short-term electricity price for Nord Pool, California, and Ontario power markets. The experiment results showed that the forecasting error has decreased 1\% 6\% compared with other grey models. Although grey theory needs very little data, it is more suitable to forecast linear data than nonlinear data. Wang et al. [10] used a combined model based on seasonal adjustment and chaotic theory to forecast electricity price of Austria power market. A phase space was reconstructed from the time series representing the data's chaotic characteristics. Particle swarm optimization (PSO) algorithm was also employed to determine the parameters of chaotic system. The forecasting performance illustrated that the combined model is better than single models. The most popular approach is the time series algorithm; stationary time series models such as autoregressive (AR) [11], dynamic regression and transfer function [1214], and autoregressive integrated moving average (ARIMA) and nonstationary time series models like generalized autoregressive conditional heteroskedastic (GARCH) have been proposed for this purpose. Specifically, Contreras et al. [15] applied ARIMA model to predict the next-day electricity price of the Spanish electricity market. However, when facing a particularly big fluctuation time sequence, especially heteroscedastic time series prediction whose variance changes over time, ARIMA model does not work well. Comparing with the traditional ARIMA model, GARCH model can commendably predict the condition heteroscedastic time series, so it is widely applied in the field of finance. Garcia et al. [16] presented GARCH model to forecast hourly prices in the electricity markets of Spain and California.

Although time series models like ARIMA and GARCH are nonlinear predictors that can meet the condition of power price, behavior of price signal may not be completely captured by the time series techniques. To solve this problem, some other artificial methods can be proposed. Artificial intelligence (AI) approaches such as neural network and support vector machine (SVM), which have been successfully applied in load forecast, are also suitable for price forecast [17]. Unlike most of the traditional neural network models which implement the empirical risk minimization principle, SVM adopts the structural risk minimization principle which seeks to minimize an upper bound of the generalization error rather than minimize the training error [18]. SVM possesses a concise mathematical form and good generalization ability; it can well solve the practical problems of the small sample size, nonlinearity, high dimension, and local minimum point.

For the forecasting models, single model has its own independent information of the electric power system, and the proper selection of the individual model can lessen the systemic information loss. Although traditional single time series models like AR, MA, and ARMA are suitable to forecast stable and linear sequences and ARIMA model can be used to forecast nonstationary and nonlinear time series, the forecasting performance is not satisfied. In addition, the fluctuation of electricity price depends on many complicated random factors in its evolution process, and the combined forecasting model can make full use of the characteristic of single models and reduce the sensitivity of the poorer prediction method. Therefore, an optimized combined model by ant colony optimization algorithm (ACO) based on GARCH model and SVM model is proposed to predict electricity price. Section 2 introduces the combined forecasting model theory, GARCH model, SVM model, and an intelligent optimization algorithm called ACO. In Section 3, a case study about forecasting electricity price of New South Wales in Australia is demonstrated. Correspondingly, GARCH model and SVM model are utilized to forecast electricity price. Then, ACO algorithm can determine the weight coefficients. The results have shown that the optimized combined method is more reliable than the individual forecasting models. Section 4 concludes this study.

So, it is still an essential need to find more accurate and robust approaches for daily electricity price prediction and an overall assessment of the price-forecasting algorithms is still required.

\section{Materials and Methods}

2.1. The Optimized Combined Forecasting Model by ACO Algorithm. The combined forecasting theory states that if there exist $M$ kinds of models to solve a certain forecasting problem, with properly selected weight coefficients, several forecasting methods' results can be added up. Assume that $y_{t}(t=1,2, \ldots, L)$ is the actual time series data, $L$ is the number of sample points, and $\mid \omega_{i}(i=1,2, \ldots, M)$ is the weight coefficient for the $i$ th forecasting model, the mathematical model of the combined forecasting model can be expressed as

$$
\begin{gathered}
y_{t}=\sum_{i=1}^{M} \omega_{i}\left(y_{i t}+e_{i t}\right), \quad t=1,2, \ldots, L, \\
\widehat{y}_{t}=\sum_{i=1}^{M} \widehat{\omega}_{i} \widehat{y}_{i t}, \quad t=1,2, \ldots, L,
\end{gathered}
$$


where $\widehat{\omega}_{i}$ is the estimated value of $\omega_{i}$ and $\widehat{y}_{t}$ is the combined forecasting value.

Determination of the weight coefficients for each individual model is the key step in constructing of a combined forecasting model. This can be achieved by solving an optimization problem which minimizes the mean absolute percentage error (MAPE) for the combined model. This objective function using can be expressed as

$$
f=\sum_{t=1}^{L} \frac{\left|y_{t}-\hat{y}_{t}\right|}{y_{t}},
$$

st $\sum_{i=1}^{M} \omega_{i}=1,0 \leq \omega_{i} \leq 1, i=1,2, \ldots, M$

The optimization process can employ ACO algorithm to minimize objective function $f$.

2.2. GARCH Model. The ARCH model was initially introduced by Engle [19], in order to account for the presence of heteroscedasticity in economic and financial time series. In an $\mathrm{ARCH}(q)$ process, the volatility at time $t$ is a function of the observed data at $t-1, t-2, \ldots, t-q$. But in the practical application, the ARCH model often needs a very long condition variance equation, and in order to avoid negative variance parameter estimation, it often needs to forcibly demand a fixed hysteresis structure [20]. On this occasion, in order to make ARCH model have long-term memorizing ability and more flexible lag structure, it is essential to extend ARCH model. Later, Bollerslev [20] introduced the generalized ARCH (GARCH) process, where conditional variance not only depends on the squared error term, but also depends on the previous conditional variance [21]. A GARCH process of orders $p$ and $q$, denoted as GARCH ( $p$, $q)$, (GARCH model is used in EVIEWS soft) can be described as follows [22]:

$$
\begin{aligned}
U_{t} & \mid F_{t-1} \sim N\left(0, h_{t-1}\right), \\
h_{t} & =\alpha_{0}+\alpha_{1} U_{t-1}^{2}+\cdots+\alpha_{q} U_{t-q}^{2}+\beta_{1} h_{t-1}+\cdots+\beta_{q} h_{t-q} \\
& =\alpha_{0}+\sum_{i=1}^{q} \alpha_{i} U_{t-i}^{2}+\sum_{j=1}^{p} \beta_{j} h_{t-j},
\end{aligned}
$$

where $q>0, \alpha_{0}>0$, and $\alpha_{i} \geq 0$ for $i=1,2, \ldots q$ and $\beta_{j} \geq 0$ for $j=1,2, \ldots p$. Again, the conditions $\alpha_{0}>0, \alpha_{i} \geq 0$, and $\beta_{j} \geq 0$ are needed to guarantee that the conditional variance $h_{t} \geq 0$. This study uses GARCH $(1,1)$ model and the $(1,1)$ in parenthesis indicates that one length of $\mathrm{ARCH} \log \left(\alpha_{1}\right)$ and one length of GARCH $\log \left(\beta_{1}\right)$ are used.

2.3. Support Vector Machine (SVM). According to the statistical learning theory and development, SVM is based on structural risk minimization (SRM) principle to minimize the generalization error; the general view is to minimize the training error and at the same time minimize the model complexity, which has become the cornerstone of modern intelligent algorithm [23]. The characteristics of SVM make it a good candidate model to apply in predicting defect-prone modules as such conditions are typically encountered.
SVM principle is as follows: given the training sample $\left\{\left(x_{i}, y_{i}\right): x_{i} \in R^{d}, y_{i} \in\{-1,+1\}\right\}_{i=1}^{N}$, then the two-class pattern recognition problem can be cast as the primal problem of finding a hyperplane: $w^{\mathrm{T}} x+b=1$, where $w$ is a $d$-dimensional normal vector, such that these two classes can be separated by two margins both parallel to the hyperplane; that is, for each $x_{i}, i=1,2, \ldots, N$

$$
\begin{gathered}
w^{\mathrm{T}} x_{i}+b \geq 1+\zeta_{i} \quad \text { for } y_{i}=+1, \\
w^{\mathrm{T}} x_{i}+b \leq-1-\zeta_{i} \quad \text { for } y_{i}=-1,
\end{gathered}
$$

where $\zeta_{i} \geq 0, i=1,2, \ldots, N$, are slack variables and $b$ is the bias. This primal problem can easily be cast as the following quadratic optimization problem [24]:

$$
\begin{aligned}
& \text { Minimizing } \quad \psi(w, \zeta)=\frac{1}{2}\|w\|^{2}+C\left(\sum_{i=1}^{N} \zeta_{i}\right) \\
& \text { subject to } y_{i}\left[\left(w \cdot x_{i}\right)-b\right] \geq 1-\zeta_{i}
\end{aligned}
$$

where $\zeta=\left(\zeta_{1}, \zeta_{2}, \ldots, \zeta_{N}\right)$

The objective of a SVM is to determine the optimal $w$ and optimal bias $b$ such that the corresponding hyperplane separates the positive and negative training data with maximum margin and it produces the best generation performance. This hyperplane is called an optimal separating hyperplane [25].

2.4. Ant Colony Optimization Algorithm (ACO). The ant colony optimization (ACO), developed by Dorigo et al. [26, 27], is a metaheuristic method that aims to find approximate solutions to optimization problems. The original idea behind ant algorithms came from the observations of the foraging behavior of ants and stigmergy. Stigmergy is a term that refers to the indirect communication amongst a selforganizing emergent system by individuals modifying their local environment [28]. The detailed steps of ACO algorithm [29] can be described as follows.

Step 1 (initializing some parameters). The algorithm starts by initializing some specific variables such as the maximum of allowed iterations NCHO and the number of ants ANT and the initial point which is randomly selected.

Step 2 (dividing the definition domain and grouping the ants). According to (6), the definition domain is divided into subregions. Each subregion is defined as (7). For all $i$ ( $i=$ $1,2, \ldots$, part $), m$ ants are assigned in every subregion randomly. Correspondingly, each ant is denoted by ant $\mathrm{t}_{i j}(i=$ $1,2, \ldots$, part $j=1,2, \ldots, m)$

$$
\begin{aligned}
l_{i} & =\frac{\max _{i}-\min _{i}}{\operatorname{part}}, \\
\operatorname{grp}_{i} & =\left[\min _{i}+l_{i} \cdot(j-1), \min _{i}+l_{i} \cdot j\right],
\end{aligned}
$$

where $i \in[1, d], j \in[1$, part $]$, and $d$ is the dimension of the independent variables. 
Step 3 (initializing the pheromone concentration). Each ant has pheromone, and the initial pheromone concentration of $j$ th ant in $i$ th subregion is defined as

$$
\tau_{i j}=e^{\left(-f\left(X_{i j}\right)\right)} \quad i=1,2, \ldots, \text { part }, \quad j=1,2, \ldots, m,
$$

where $X_{i j}$ represents the location of the $j$ th ant in $i$ th subregion and $f\left(X_{i j}\right)$ is the objective function. If the pheromone is greater, the function value is smaller.

Step 4 (determining the elite ant (the optimal ant)). The pheromone of the elite ant in each group (the local optimal ant) can be defined as follows:

$$
\tau \max _{i}=\max \left(\tau_{i 1}, \tau_{i 2}, \ldots, \tau_{i j}\right),
$$

where $i=1,2, \ldots$, part and $j=1,2, \ldots, m$.

Then based on (10), the pheromone of the global elite ant (the global optimal ant) can be got

$$
\tau_{k l}=\max \left(\tau \max _{1}, \tau \max _{2}, \ldots, \tau \max _{i}\right),
$$

where $i=1,2, \ldots$, part, $k \in[1$, part $]$, and $l \in[1, m]$. So $k$ th ant in $l$ th subregion is the global elite ant, and $X_{k l}$ is the location of the global elite ant.

Step 5 (updating the ant's location of each group). If $\mid D_{i}-$ $D_{j} \mid \geq\left(\left|D_{\max }-D_{\min }\right| / 10\right)$, then

$$
D_{i}^{\prime}= \begin{cases}\tau_{i} D_{i}+\alpha_{1} \frac{1-\tau_{i}}{\tau_{j}}\left(D_{j}-D_{i}\right), & \text { if } \alpha_{1}<0.5 \\ \tau_{i} D_{i}+\alpha_{1}\left(1-\tau_{i}\right) D_{j}, & \text { otherwise. }\end{cases}
$$

If $\left|D_{i}-D_{j}\right|<\left(\left|D_{\max }-D_{\min }\right| / 10\right)$, then let $\mu=\left|D_{i}-D_{j}\right|$

$$
D_{i}^{\prime}= \begin{cases}D_{i}+\mu \cdot \delta \cdot \rho, & \rho<\rho_{0} \\ D_{i}-\mu \cdot \delta \cdot \rho, & \rho>\rho_{0},\end{cases}
$$

where in this generation $D_{j}(j \in[1, m])$ is the location of the elite ant, $\tau_{j}$ is the pheromone of the elite ant, $D_{i}(i \in[1, m])$ is the location of the common ant, and $\tau_{i}$ is the pheromone of the common ant. $D_{i}^{\prime}$ is the location of the common ant after updating, $\alpha_{1} \in(-1,1), \rho \in(0,1), \rho_{0} \in(0,1)$, and $\delta \in 0.1 \times$ $\operatorname{rand}(1)$.

Step 6 (get the current global optimal solution). Get the current global optimal solution $f_{\text {opt }}\left(X_{i j}\right)(i=[1$, part $], j \in$ $[1, m])$ based on the new location of the ants and update the pheromone of each ant in each subregion based on

$$
\tau_{i j}=\rho \tau_{i j}+e^{-f\left(X_{i j}\right)}
$$

Step 7. $n c h o=n c h o+1$, if $n c h o>N C H O$, go to Step 9, otherwise go to Step 8.

Step 8. If $f_{\text {opt }}\left(X_{i j}\right)(i \in[1$, part $], j \in[1, m])$ is less than the given solution error condition $10^{-2}$, then go to Step 9 , otherwise go to Step 4.

Step 9. Output $n c h o$ and $f_{\text {opt }}\left(X_{i j}\right)(i \in[1$, part $], j \in[1, m])$.

\section{A Case Study}

3.1. Evaluation Criterion of Forecasting Performance. Two loss functions can be served as the criteria to evaluate the prediction performance relative to electricity price value, including mean absolute error (MAE) and mean absolute percentage error (MAPE); the forecasting effect is better when the loss function value is smaller. The two loss functions are expressed as follows:

$$
\begin{aligned}
\mathrm{MAE} & =\frac{1}{L} \sum_{t=1}^{L}\left|y_{t}-\widehat{y}_{t}\right|, \\
\mathrm{MAPE} & =\frac{1}{L} \sum_{t=1}^{L} \frac{\left|y_{t}-\widehat{y}_{t}\right|}{y_{t}} \times 100 \%,
\end{aligned}
$$

where $y_{t}$ and $\hat{y}_{t}$ represent actual and forecasting electricity price at time and the value of $L$ in our study is 48 .

3.2. Simulation and Analysis of Results. The proposed optimized combined method is tested using a case study about forecasting electric price of New South Wales, Australia. The detailed forecasting procedure can be seen in Figure 1. The electricity price data were collected on a half-hourly basis ( 48 data points per day, starting from 0:00 AM to 23:30 PM) for 5 Mondays of electricity price values from February 12, 2012, to March 11, 2012, to predict 1 Monday of electricity price in March 18, 2012. The actual electricity price data can be seen in Figure 2.

GARCH model is operated in EVIEWS soft to forecast electricity price series of New South Wales. Before doing work, this paper judges whether we can apply GARCH (1, 1) model by ARCH Lagrangian Multipliers Test (ARCH LM Test). At the beginning, the least square method is used to estimate electricity price data; then, $\mathrm{ARCH}$ test is performed over the residuals by observing the values of the F-statistics, which is not strong. Figure 1 illustrates these procedures. Let the confidence level $\alpha$ be set to 0.05 . If probability $P$ is less than 0.05 , the residual sequences will have $\mathrm{ARCH}$ effect. In other words, it is suitable to use GARCH model. Specifically, the results of the ARCH test are presented in Table 1 and it is found that GARCH $(1,1)$ model can be applied to forecast electricity price.

The SVM model, which is skilled in dealing with small simple and nonlinear data, will be used in the next step. The number of actual data is 240 (5 Mondays), so the number of $w$ is 240 and the detailed values of $w$ are seen in Figure 3. The value of the bias $b=-0.2874$. After simulation, the forecasted values can be presented in Table 2 .

However, single traditional model has some limitations which cannot present the characteristics of data well. Therefore, many combined models usually are used to forecast electricity price in power system. In this study, an optimized combined model based on GARCH and SVM can be proposed. Correspondingly, ACO algorithm is presented to optimize and determine the weight coefficients. In ACO algorithm, the experiment uses some parameters as follows: $d=1, A N T=36, N C H O=100, \varphi=0.5$, and $\rho=$ 0.5 . In fact, we are not only interested in simulation of ant 


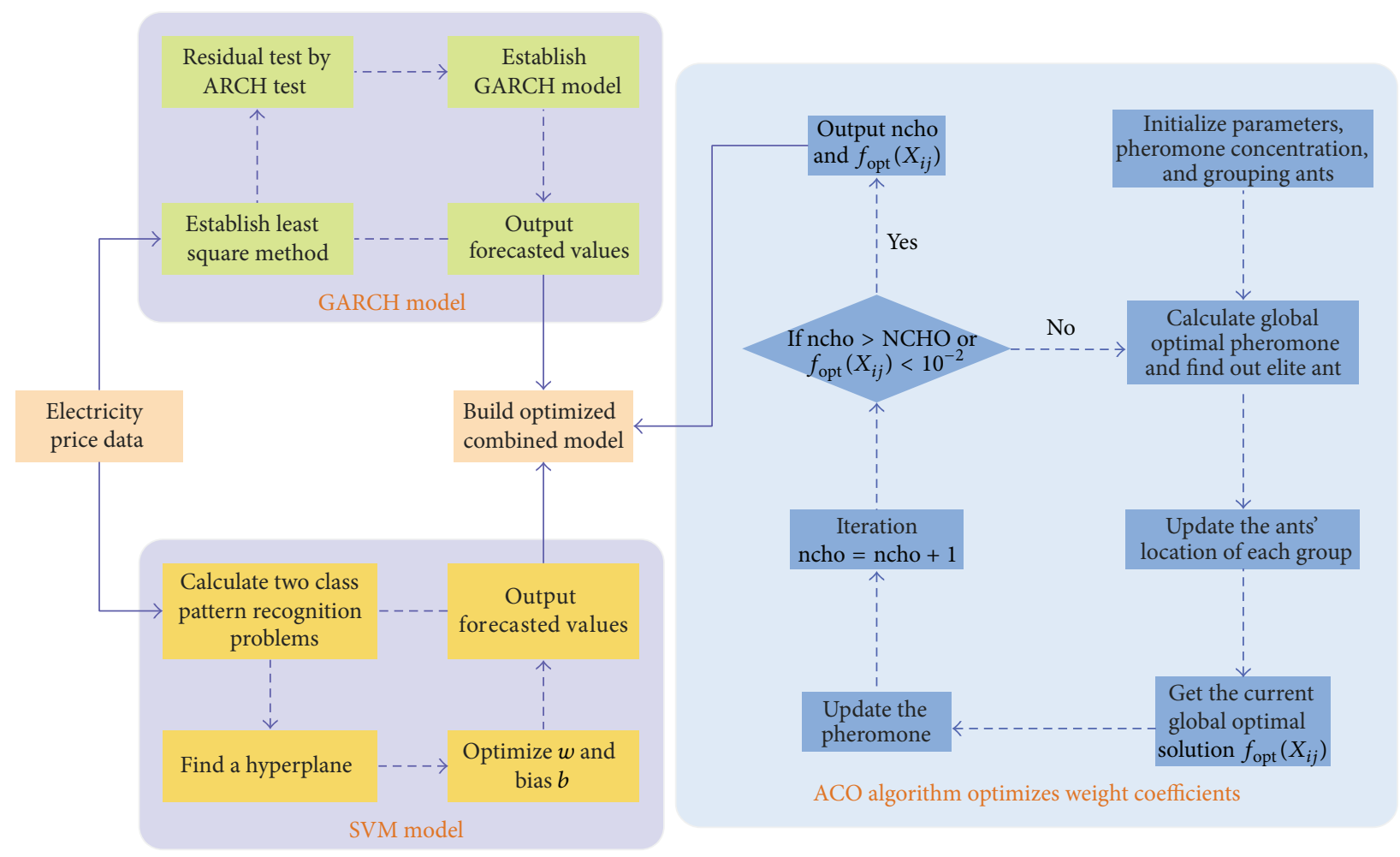

FIGURE 1: Flow chart of the optimized combined model.

TABLE 1: ARCH test.

\begin{tabular}{lccc}
\hline & \multicolumn{3}{c}{ ARCH test } \\
\hline$F$-statistic & 1127.448 & Probability & 0.000000 \\
Obs $^{*} R$-squared & 229.0899 & Probability & 0.000000 \\
\hline$R$-squared & 0.798223 & Mean dependent variance & 25.75343 \\
Adjusted $R$-squared & 0.797517 & S.D. dependent variance & 45.27648 \\
S.E. of regression & 20.37368 & Akaike info criterion & 8.873309 \\
Log likelihood & -1271.320 & Schwarz criterion & 8.898811 \\
Durbin Watson stat & 2.178122 & Probability $(F$-statistic $)$ & 0.000000 \\
\hline
\end{tabular}

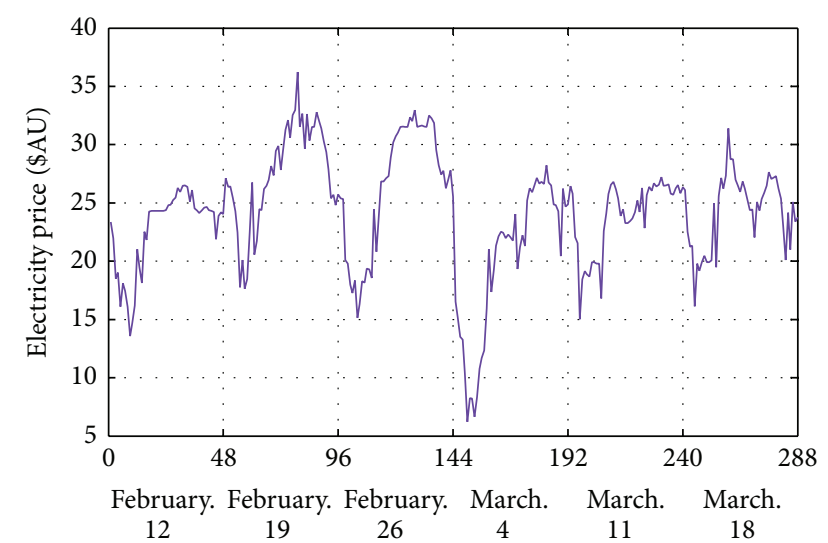

FIgUre 2: The actual electricity price data of six Mondays in New South Wales of Australia in 2012. colonies, but also in the use of artificial ant colonies as a one-dimensional optimization tool. The objective function is $f=\sum_{t=1}^{L}\left(\left|y_{t}-\widehat{y}_{t}\right| / y_{t}\right)$ in (2) and $\widehat{y}_{t}=\sum_{i=1}^{M} \widehat{\omega}_{i} \hat{y}$. Its aim is to optimize $\widehat{\omega}_{i}$ so as to minimize the objective function $f$. The algorithm would stop when the number of the maximum iteration is 100 . Through simulation and calculation, we can get $\widehat{\omega}_{1}=0.8058, \widehat{\omega}_{2}=0.1942$. So the estimated values of the combined model are written as $\hat{y}=0.8058 \times \widehat{y}_{1}+0.1942 \times \widehat{y}_{2}$. The concrete values can be seen in Table 2 .

In addition, we produce whisker plot with three boxes which have lines at the lower quartile, median, and upper quartile values of prediction electricity price value by GARCH $(1,1)$, SVM model, and the optimized combined model in Figure 4. It is easy to find that each of the boxes includes a notch in the position of the median value. The 


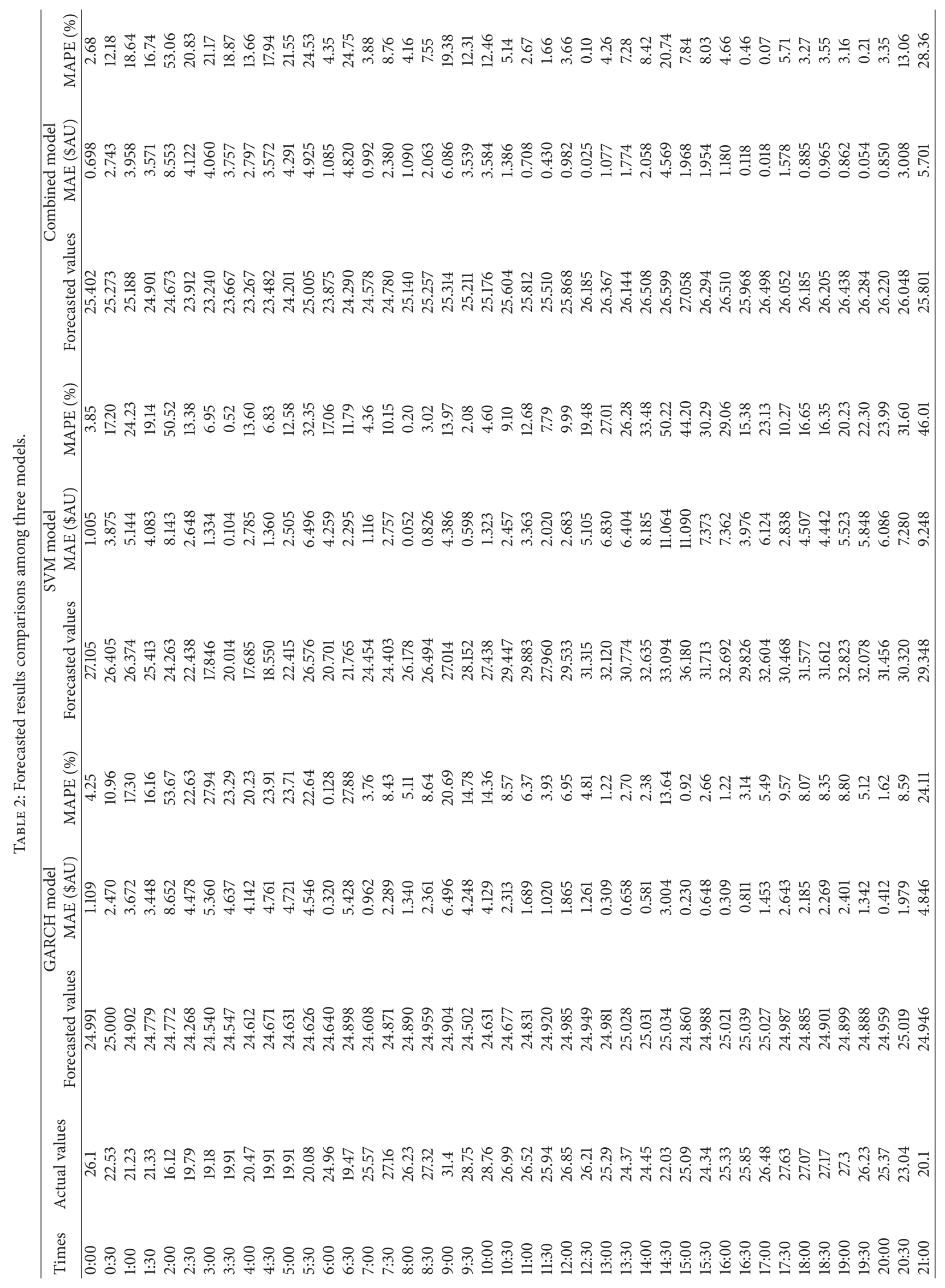




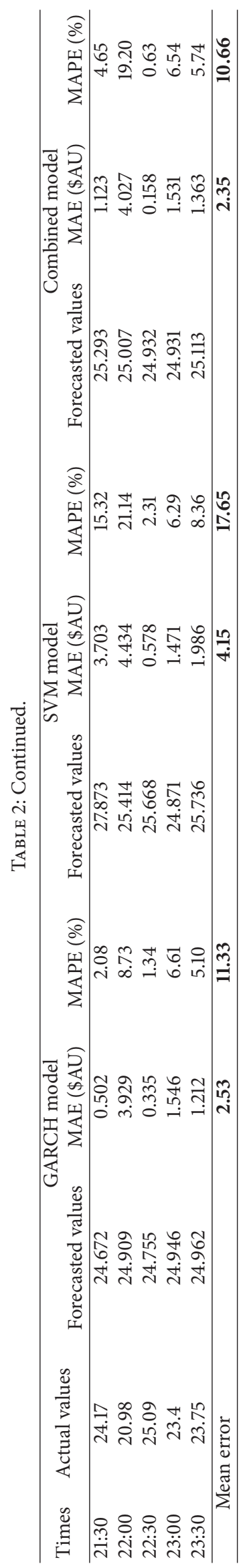




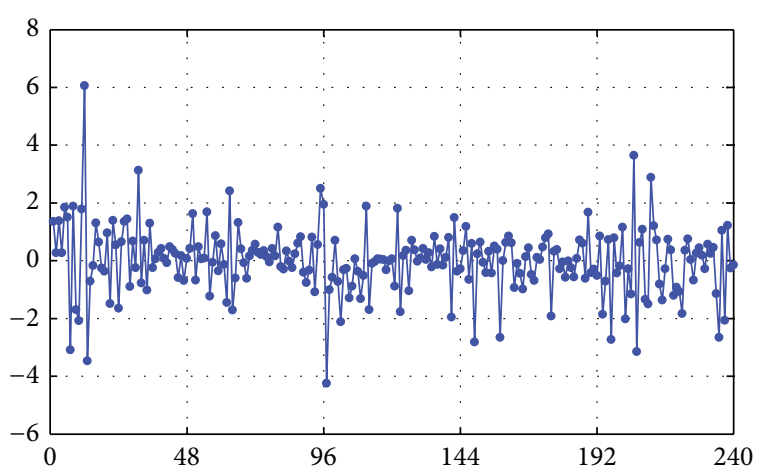

FIGURE 3: The detailed values of $w$ in the hyperplane.

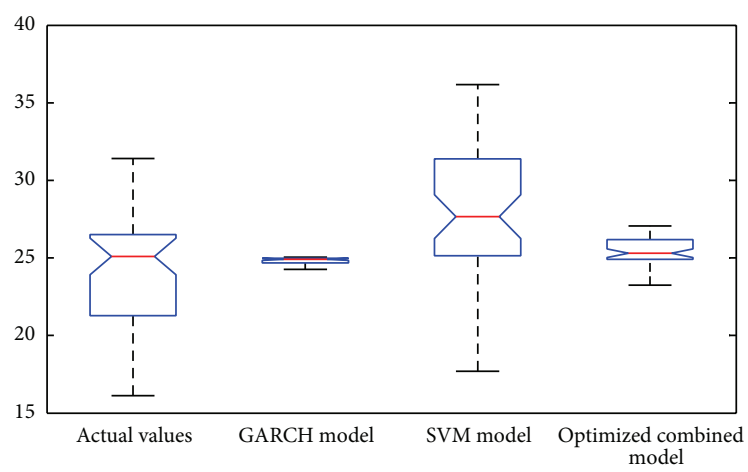

Figure 4: Box graphs of the actual values and the forecasted values by three models.

mean values of GARCH model and the optimized combined model are closer to the actual values.

In order to further illustrate the validity of the combined model, two kinds of errors including MAE and MAPE of GARCH model and SVM model have been listed in Table 2. It is clear that MAE and MAPE using GARCH $(1,1)$ model or SVM model are higher than the combined model. Although the forecasted values of SVM model are close to the actual values before 7:00, the differences are great large after 7:00. Also, it has been observed that the proposed optimized combined model leads to 0.18 \$AU reductions in total mean MAE and $0.67 \%$ reductions in total mean MAPE, respectively, in comparison with traditional individual GARCH $(1,1)$ model and results in $1.8 \$ A U$ reductions in total mean MAE and approximately $7 \%$ reductions in total mean MAPE, respectively, compared to the single SVM model. Consequently, the results obtained from the optimized combined model agree with the actual electricity price exceptionally well. In other words, the forecasting model using optimized combined model can yield better results than using GARCH $(1,1)$ and SVM model.

\section{Conclusions}

The development of industries, agriculture, and infrastructure depends on the electric power system; electricity consumption is relative to modern life; consumers want to minimize their electricity costs and producers expect to maximize their profits and avoid volatility; therefore, it is important for accurate electricity price prediction.

There are four advantages of the optimized combined method. At first, the optimized combined model by ACO algorithm based on GARCH $(1,1)$ model and SVM method for forecasting half-hourly real-time electricity prices of New South Wales creates commendable improvements that are relatively satisfactorily for current research. Second, the individual model has its own independent characteristic in the power system. The proper selection of traditional single model can lessen the systemic information loss. The optimized combined forecasting model can make full use of single models and it is less sensitive to the poorer forecasting approach. On the basis there is no doubt that the improved combined forecasting model performs better than conventional single model. Third, an intelligent optimization algorithm called ant colony optimization is used to determine the weight coefficients. Finally, the optimized combined model is essentially automatic and does not require to make complicated decision about the explicit form of models for each particular case. The combined forecasting procedure gives the minimum MAE and MAPE.

The final result is that the optimized model has high prediction accuracy and good prediction ability. With proper characteristic selection, redundant information is overlooked or even eliminated; a more efficient and straightforward model is got. To sum up, it is clear that the improved combined model is more effective than the existing individual models for the electricity price forecasting.

\section{Conflict of Interests}

The authors declare that there is no conflict of interests regarding the publication of this paper.

\section{Acknowledgments}

The authors would like to thank the Natural Science Foundation of P. R. of China (61300230), the Key Science and Technology Foundation of Gansu Province (1102FKDA010), Natural Science Foundation of Gansu Province (1107RJZA188), and the Fundamental Research Funds for the Central Universities for supporting this research.

\section{References}

[1] S. K. Aggarwal, L. M. Saini, and A. Kumar, "Electricity price forecasting in deregulated markets: a review and evaluation," International Journal of Electrical Power \& Energy Systems, vol. 31, no. 1, pp. 13-22, 2009.

[2] D. W. Bunn, "Forecasting loads and prices in competitive power markets," Proceedings of the IEEE, vol. 88, no. 2, pp. 163-169, 2000.

[3] R. E. Abdel-Aal, "Modeling and forecasting electric daily peak loads using abductive networks," International Journal of Electrical Power \& Energy Systems, vol. 28, no. 2, pp. 133-141, 2006.

[4] P. Mandal, T. Senjyu, N. Urasaki, and T. Funabashi, "A neural network based several-hour-ahead electric load forecasting 
using similar days approach," International Journal of Electrical Power \& Energy Systems, vol. 28, no. 6, pp. 367-373, 2006.

[5] N. Amjady and M. Hemmati, "Energy price forecasting: problems and proposals for such predictions," IEEE Power and Energy Magazine, vol. 4, no. 2, pp. 20-29, 2006.

[6] R. Lapedes and R. Farber, "Nonlinear signal processing using neural networks:prediction and system modeling," Tech. Rep. LA-VR87-2662, Los Alamos National Laboratory, Los Alamos, NM, USA, 1987.

[7] J. V. E. Robert, The Application of Neural Networks in the Forecasting of Share Prices, Finance and Technology Publishing, 1996.

[8] A. I. Arciniegas and I. E. Arciniegas Rueda, "Forecasting shortterm power prices in the Ontario Electricity Market (OEM) with a fuzzy logic based inference system," Utilities Policy, vol. 16, no. 1, pp. 39-48, 2008.

[9] M. L. Lei and Z. R. Feng, "A proposed grey model for short-term electricity price forecasting in competitive power markets," International Journal of Electrical Power \& Energy Systems, vol. 43, no. 1, pp. 531-538, 2012.

[10] J. Wang, H. Lu, Y. Dong, and D. Chi, "The model of chaotic sequences based on adaptive particle swarm optimization arithmetic combined with seasonal term," Applied Mathematical Modelling, vol. 36, no. 3, pp. 1184-1196, 2012.

[11] O. B. Fosso, A. Gjelsvik, A. Haugstad, B. Mo, and I. Wangensteen, "Generation scheduling in a deregulated system. the norwegian case," IEEE Transactions on Power Systems, vol. 14, no. 1, pp. 75-80, 1999.

[12] H. Zareipour, C. A. Cañzares, K. Bhattacharya, and J. Thomson, "Application of public-domain market information to forecast Ontario's wholesale electricity prices," IEEE Transactions on Power Systems, vol. 21, no. 4, pp. 1707-1717, 2006.

[13] F. J. Nogales, J. Contreras, A. J. Conejo, and R. Espínola, "Forecasting next-day electricity prices by time series models," IEEE Transactions on Power Systems, vol. 17, no. 2, pp. 342-348, 2002.

[14] F. J. Nogales and A. J. Conejo, "Electricity price forecasting through transfer function models," Journal of the Operational Research Society, vol. 57, no. 4, pp. 350-356, 2006.

[15] J. Contreras, R. Espínola, F. J. Nogales, and A. J. Conejo, "ARIMA models to predict next-day electricity prices," IEEE Transactions on Power Systems, vol. 18, no. 3, pp. 1014-1020, 2003.

[16] R. C. Garcia, J. Contreras, M. van Akkeren, and J. B. C. Garcia, "A GARCH forecasting model to predict day-ahead electricity prices," IEEE Transactions on Power Systems, vol. 20, no. 2, pp. 867-874, 2005.

[17] B. R. Szkuta, L. A. Sanabria, and T. S. Dillon, "Electricity price short-term forecasting using artificial neural networks," IEEE Transactions on Power Systems, vol. 14, no. 3, pp. 851-857, 1999.

[18] F. E. H. Tay and L. Cao, "Application of support vector machines in financial time series forecasting," Omega, vol. 29, no. 4, pp. 309-317, 2001.

[19] R. F. Engle, "Autoregressive conditional heteroscedasticity with estimates of the variance of United Kingdom inflation," Econometrica, vol. 50, no. 4, pp. 987-1007, 1982.

[20] T. Bollerslev, "Generalized autoregressive conditional heteroskedasticity," Journal of Econometrics, vol. 31, no. 3, pp. 307327, 1986.

[21] C.-H. Tseng, S.-T. Cheng, and Y.-H. Wang, "New hybrid methodology for stock volatility prediction," Expert Systems with Applications, vol. 36, no. 2, pp. 1833-1839, 2009.
[22] R. F. Engle, "Autoregressive conditional heteroscedasticity with estimates of the variance of United Kingdom inflation," Econometrica, vol. 50, no. 4, pp. 987-1007, 1982.

[23] D. Niu, D. Liu, and D. D. Wu, "A soft computing system for day-ahead electricity price forecasting," Applied Soft Computing Journal, vol. 10, no. 3, pp. 868-875, 2010.

[24] C. Cortes and V. Vapnik, "Support-vector networks," Machine Learning, vol. 20, no. 3, pp. 273-297, 1995.

[25] H.-J. Lin and J. P. Yeh, "Optimal reduction of solutions for support vector machines," Applied Mathematics and Computation, vol. 214, no. 2, pp. 329-335, 2009.

[26] M. Dorigo, V. Maniezzo, and A. Colorni, "Ant system: optimization by a colony of cooperating agents," IEEE Transactions on Systems, Man, and Cybernetics B: Cybernetics, vol. 26, no. 1, pp. 29-41, 1996.

[27] M. Dorigo and T. Stützle, Ant Colony Optimization, MIT Press, Cambridge, Mass, USA, 2004.

[28] M. J. Meena, K. R. Chandran, A. Karthik, and A. V. Samuel, "An enhanced ACO algorithm to select features for text categorization and its parallelization," Expert Systems with Applications, vol. 39, no. 5, pp. 5861-5871, 2012.

[29] Q. Y. Li, Q. B. Zhu, and W. Ma, "Grouped ant colony algorithm for solving continuous optimization problems," Computer Engineering and Applications, vol. 46, no. 30, pp. 46-49, 2010. 


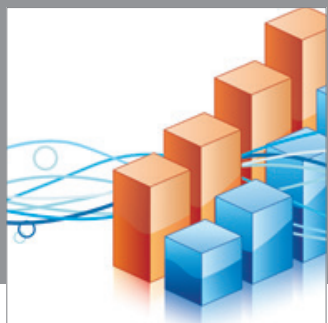

Advances in

Operations Research

mansans

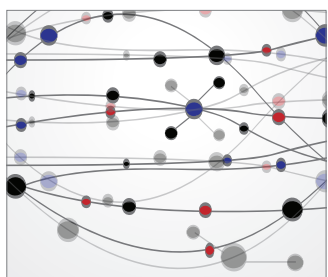

The Scientific World Journal
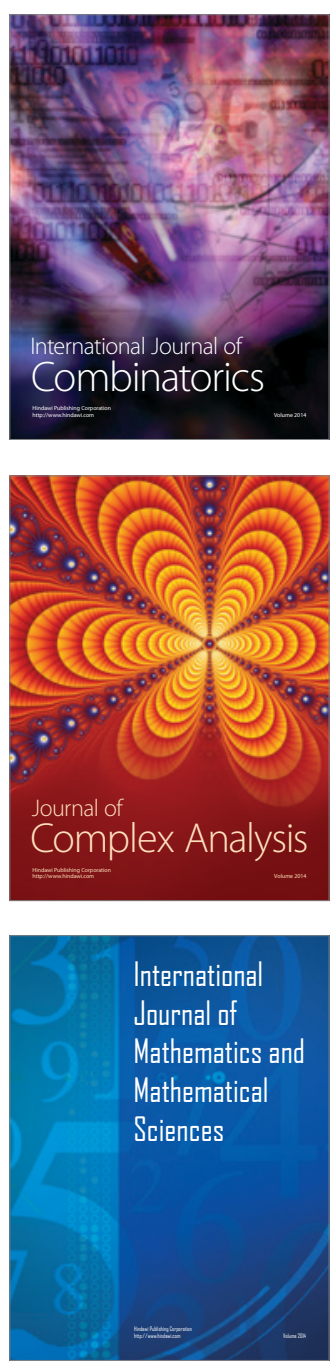
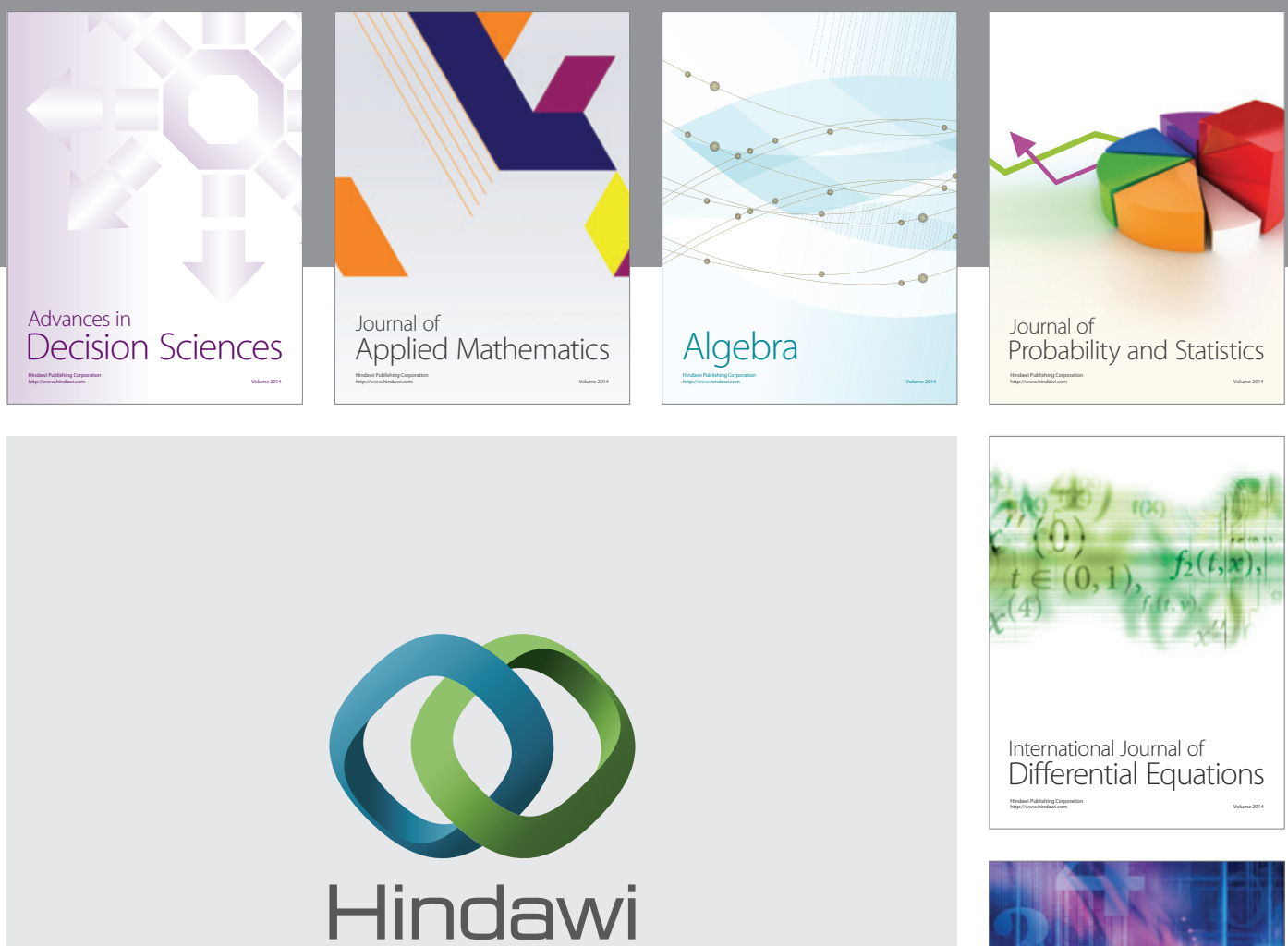

Submit your manuscripts at http://www.hindawi.com
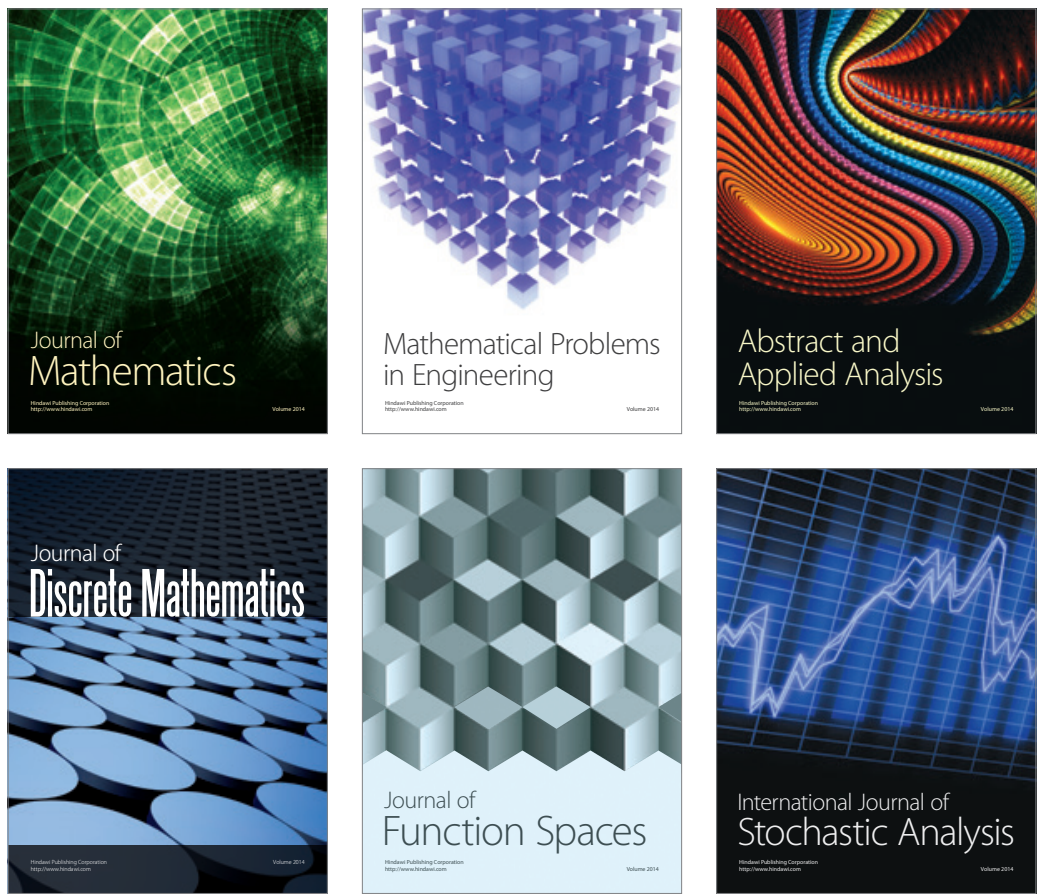

Journal of

Function Spaces

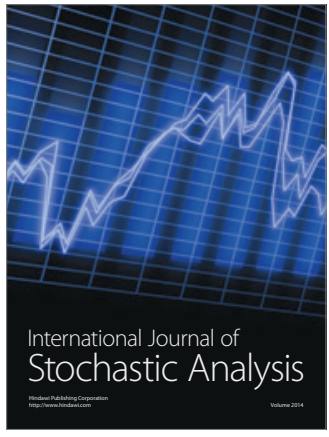

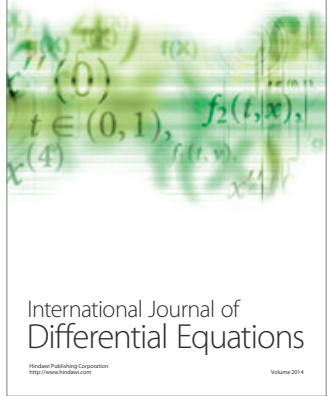
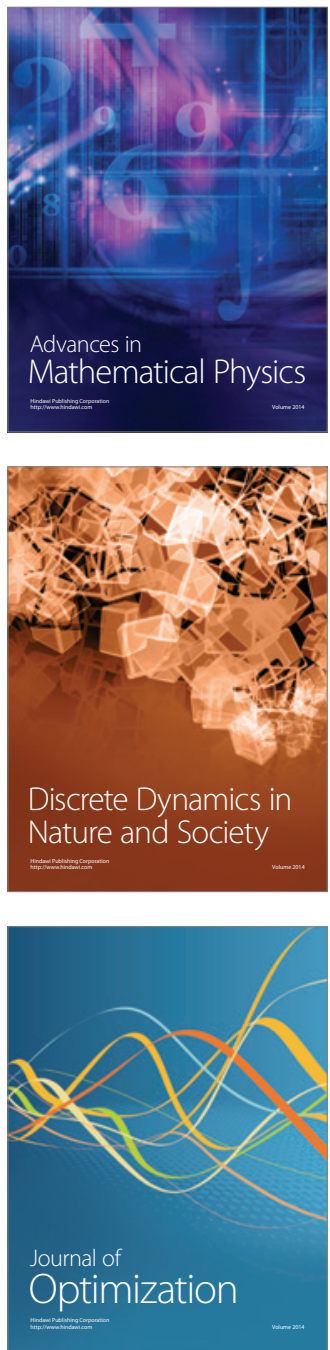